\title{
Les jonctions intercellulaires endothéliales dans la formation des vaisseaux
}

\author{
Philippe Huber
}

Laboratoire de Physiopathologies Vasculaires. Unité CEA - Inserm - Université Joseph Fourier. CEA-Grenoble iRTSV-LAPV-U882, 17 rue des Martyrs 38054 Grenoble. phuber@cea.fr Mots-clés : adhérence cellulaire, cadhérines, angiogenèse, perméabilité vasculaire

\section{Endothelial cell-cell junctions in vessel formation}

The endothelium, lining the inner side of all vessel types, is constituted of a monolayer of endothelial cells with cobblestone morphology. Endothelial cell-cell contacts contain numerous transmembrane adhesive proteins that are either clustered in junctional structures or located along the intercellular cleft. These proteins promote cell-cell adhesion and control vascular permeability to fluids and molecules, as well as transmigration of various types of leukocytes. In addition, recent findings showed that constituents of the junctions might be part of the vascular invasion machinery by activating cell protrusions. Such activities may thus be considered as markers of pathological angiogenesis or targets of antiangiogenic therapy.

\section{Résumé}

L'endothélium, localisé sur la face interne de tous types de vaisseaux, est constitué d'une monocouche pavimenteuse de cellules endothéliales. La zone de contact intercellulaire endothéliale contient plusieurs protéines transmembranaires à activité adhésive qui sont soit inclues dans des structures jonctionnelles spécifiques, soit localisées le long de la zone de contact. Ces protéines sont essentielles à l'adhérence intercellulaire et au contrôle de la perméabilité vasculaire aux fluides, aux molécules et à la transmigration de plusieurs types de 
globules blancs. Récemment, il a été montré que des constituants des jonctions pouvaient également être impliqués dans l'activité protrusive des cellules permettant l'invasion cellulaire. Ces activités peuvent donc être considérées comme des cibles pour des thérapies antiangiogéniques.

\section{Introduction}

La zone de recouvrement entre deux cellules endothéliales contient un domaine de contact intercellulaire dans lequel les cellules endothéliales sont séparées de 10 à $20 \mathrm{~nm}$. Ce domaine de contact contient également des structures jonctionnelles complexes appelées « jonction adhérente », « jonction serrée » et « jonction de communication » (Wallez \& Huber, 2008). Il est important de noter que ces structures sont très dynamiques et qu'elles sont soumises à des régulations multiples. En outre, les leucocytes transmigrent du sang vers les tissus (pour le système sanguin) ou des tissus vers la lymphe (pour le système lymphatique) par des voies paracellulaires (aux jonctions) ou éventuellement transcellulaires. La transmigration leucocytaire est un processus rapide et régulé au cours duquel le leucocyte se faufile à travers la jonction (diapédèse). Ce phénomène s’accompagne de l'ouverture puis d'une refermeture rapide de la zone jonctionnelle (Allport et al., 2000; Shaw et al., 2001; Su et al., 2002). L'endothélium est également à l'origine de la formation de nouveaux vaisseaux par un phénomène appelé «angiogenèse », dans lequel des cellules endothéliales migrent et prolifèrent, après stimulation et guidage par des cytokines angiogéniques. Ce phénomène a lieu de façon extensive au cours du développement embryonnaire, lors de réparation tissulaire ou dans l’utérus et l'ovaire au cours du cycle ovarien chez la femme. Il est également mis en jeu dans différentes pathologies comme la croissance tumorale, la rétinopathie diabétique, l'athérosclérose et l'arthrite rhumatoïde (Carmeliet \& Jain, 2000). Dans toutes ces affections, l’angiogenèse favorise la pathogenèse. 


\section{La jonction quiescente}

À l'état quiescent, l'adhérence intercellulaire est soutenue par différents types de protéines transmembranaires développant une activité adhésive homophile (permettant l'interaction avec une molécule identique localisée sur la cellule adjacente). Ces protéines sont soit localisées dans des structures jonctionnelles telles que la jonction adhérente ou la jonction serrée, soit réparties librement le long de la zone de contact (Wallez \& Huber, 2008). L’interaction forte est réalisée par la jonction adhérente qui est associée étroitement au cytosquelette d'actine. La jonction serrée, également liée mais moins fortement à l'actine, est principalement responsable de l'étanchéité jonctionnelle, du contrôle de la perméabilité sélective à ce niveau et du guidage des leucocytes lors de leur transmigration. Les molécules impliquées sont indiquées sur la Fig. 1.

\section{Les jonctions cellulaires dans l'endothélium angiogénique}

Les vaisseaux tumoraux ont un aspect différent des vaisseaux normaux: ils sont plus tortueux, plus perméables et semblent subir un remaniement permanent. Les images de microscopie électronique à balayage montrent des jonctions mal scellées et une activité filopodiale (projections cellulaires) intense, due à l'action constante de cytokines angiogéniques (Hashizume et al., 2000). Les jonctions sont perturbées par l’action de ces cytokines et nous verrons dans un premier temps par quels mécanismes moléculaires. Certaines des protéines adhésives sont également des acteurs de l'angiogenèse, et nous verrons ensuite les éléments qui permettent de mieux apprécier ce processus.

\section{Régulation des jonctions endothéliales par le VEGF}


Le vascular endothelial growth factor (VEGF ou VEGF A) est une cytokine centrale dans le déclenchement de l'angiogenèse. Il est produit par les cellules hypoxiques et interagit avec le VEGF récepteur 2 (VEGF R2) présent à la surface des cellules endothéliales, permettant ainsi la transduction de signaux intracellulaires (Eriksson \& Alitalo, 1999). Ces signaux conduisent à différentes activités cellulaires : prolifération, migration, augmentation de la perméabilité paracellulaire. L’état physiologique de la cellule et/ou la présence d'autres cytokines va moduler la réponse cellulaire et interférer avec les voies de signalisation mises en jeu par le VEGF.

Le VEGF contrôle l'activité biologique de deux protéines jonctionnelles, l'occludine et la VE-cadhérine, appartenant respectivement à la jonction serrée et à la jonction adhérente.

L’occludine est une protéine à 4 domaines transmembranaires exprimée dans l'endothélium des vaisseaux peu perméables, comme la barrière hémato-encéphalique par exemple (Hirase et al., 1997). Le VEGF agit par deux voies pour diminuer l'activité adhésive de l’occludine : un mécanisme extracellulaire faisant intervenir un activateur de protéase, l’uPA, qui va conduire à la dégradation de l'occludine (Behzadian et al., 2003), et un mécanisme intracellulaire aboutissant à l'activation de la protéine kinase $\mathrm{C}$, qui va à son tour phosphoryler l'occludine et diminuer son activité adhésive (Antonetti et al., 1999; Harhaj et al., 2006).

La VE-cadhérine est un composant majeur de l'adhérence endothéliale homophile. Cette protéine constitue le maillon transmembranaire de la jonction adhérente de tous les types d'endothélium (Dejana et al., 1999). Un complexe protéique sous-membrannaire lui est associé, permettant l'ancrage au cytosquelette mais également sa participation à plusieurs voies de signalisation intracellulaire (Wallez et al., 2006). Le VEGF en se liant à VEGF R2 va déclencher l'interaction de VEGF R2 avec le complexe de la VE-cadhérine et activer la protéine tyrosine kinase src qui est associée constitutivement à la VE-cadhérine (Lambeng et 
al., 2005). Src activée va phosphoryler directement la VE-cadhérine sur la tyrosine 685, qui devient un site d'interaction pour la kinase csk (Wallez et al., 2007). Cette interaction va avoir pour effet de limiter la prolifération cellulaire (Baumeister et al., 2005). Src active va également déclencher une cascade d’activation protéique aboutissant à la phosphorylation de la VE-cadhérine sur la sérine 665. La phospho-sérine 665 est alors reconnue par la $\beta$ arrestine, provoquant ainsi son internalisation par endocytose (Gavard \& Gutkind, 2006). Cette internalisation pourrait être responsable de la diminution de l'activité adhésive des jonctions en présence de VEGF.

\section{Activité angiogénique de constituants des jonctions endothéliales}

Le rôle important et insoupçonné de plusieurs protéines jonctionnelles dans l'angiogenèse a été révélé par les expériences d’inactivation génique chez la souris. Dans ces expériences, l’inactivation d'un gène spécifique génère des souris complètement dépourvues de la protéine codée par ce gène, ce qui permet d'en évaluer le rôle essentiel. Quatre protéines jonctionnelles, la VE-cadhérine, JAM-A, ESAM et la Pcdh12 se sont ainsi révélées être des acteurs importants ou essentiels de l'angiogenèse, en participant à des mécanismes qui sont seulement partiellement élucidés à l’heure actuelle.

Les embryons déficients en VE-cadhérine meurent à mi-gestation à la suite de défauts majeurs dans le développement vasculaire : un réseau vasculaire primitif se forme, mais l'angiogenèse est très limitée car les cellules endothéliales ont une activité invasive faible (Gory-Fauré et al., 1999). Ce phénotype est similaire à celui observé chez des souris pour lesquelles la partie cytoplasmique de la VE-cadhérine est partiellement tronquée (Carmeliet et al., 1999). Ces résultats indiquent que la VE-cadhérine a une activité angiogénique indépendante de son rôle dans la jonction quiescente. Cette activité est portée au moins partiellement par sa partie cytoplasmique. La contribution de la VE-cadhérine dans la 
formation de néo-vaisseaux a été démontrée chez l’adulte par des expériences utilisant des anticorps bloquant la VE-cadhérine qui abrogent l'angiogenèse et limitent la croissance tumorale (Liao et al., 2000; Corada et al., 2002; Liao et al., 2002). L’inactivation des gènes codant pour JAM-A, ESAM et la Pcdh12 n’est pas létale chez la souris. Cependant, des défauts plus subtiles ont été notés. Les vaisseaux déficients en JAM-A ne répondent pas à la stimulation par le FGF-2, une autre cytokine angiogénique (Cooke et al., 2006); l'angiogenèse tumorale est déficiente en absence d'ESAM (Ishida et al., 2003) et la croissance ainsi que l'angiogenèse placentaires sont ralenties lorsque la Pcdh12 est inactivée (Rampon et al., 2008). Ces résultats indiquent que les processus angiogéniques sont variables en fonction de la situation physiopathologique et que les protéines jonctionnelles participent différentiellement à ces processus.

Les mécanismes moléculaires qui sous-tendent cette activité angiogénique sont partiellement élucidés. JAM-A et la VE-cadhérine sont impliquées dans les voies d'activation mitotique mises en jeu respectivement par le FGF-2 et le VEGF : JAM-A est un maillon indispensable à l'activation des kinases ERK1/2 en réponse au FGF-2 (Naik et al., 2003a; Naik et al., 2003b), alors que la VE-cadhérine limite au contraire l'activité proliférative du VEGF (Lampugnani et al., 2003).

Un rôle direct de la VE-cadhérine et d'ESAM sur la migration cellulaire a également été évoqué. La VE-cadhérine participe au contrôle de la morphologie cellulaire et à l'activité filopodiale régie par Rho et Cdc42, deux GTPases régulant la polymérisation de l'actine et l'architecture du cytosquelette (Kouklis et al., 2003; Nelson \& Chen, 2003; Nelson et al., 2004). De façon similaire, l'absence d’ESAM diminue l'activité de Rho et limite la migration cellulaire (Wegmann et al., 2006). La VE-cadhérine est également présente aux extrémités des filopodes où elle pourrait former les premiers contacts interendothéliaux (Almagro et Gulino, résultats non publiés) et ainsi promouvoir le maillage de vaisseaux néoformés. En 
conséquence, de par leur lien étroit avec le cytosquelette, plusieurs molécules adhésives jonctionnelles sont des acteurs importants et à différents niveaux de l’angiogenèse.

\section{Conclusions}

La recherche dans le domaine de l'angiogenèse s’est beaucoup focalisée sur les cytokines angiogéniques et l'inhibition de leur action dans le cadre de stratégies angiostatiques à visée antitumorale. Ces nouvelles voies thérapeutiques connaissent un succès grandissant avec des effets spectaculaires pour différents types de cancer. Cependant, ces premières approches angiostatiques sont insuffisantes car il y a d’une part des effets secondaires non négligeables et d'autre part, parce qu'il est important de définir d'autres cibles thérapeutiques pour diversifier l'offre pharmacologique dans ce domaine. La détermination de l'activité angiogénique de protéines adhésives est donc une étape majeure dans la caractérisation de nouveaux mécanismes spécifiques de l'angiogenèse et la prospective de thérapies ciblées. C’est déjà le cas pour la VE-cadhérine qui est la cible d'une molécule en phase d'études cliniques dans le cadre d'une thérapie angiostatique et antitumorale, la combretastatine-A4 (informations du National Cancer Institute).

\section{Légende}

Fig. 1 : Principales protéines adhésives des jonctions interendothéliales. Les protéines de la famille des claudines, des JAMs, ainsi que l'occludine et ESAM sont des protéines transmembranaires formant la jonction serrée. La VE-cadhérine forme la jonction adhérente. Ces deux types de jonctions sont liées aux filaments d’actine. La Pcdh12 n’est ni associée à une structure jonctionnelle, ni liée au cytosquelette d’actine.

\section{Références}


Allport J.R., Muller W.A., and Luscinskas F.W. Monocytes induce reversible focal changes in vascular endothelial cadherin complex during transendothelial migration under flow. $J$ Cell Biol, 2000, 148, 203-216.

Antonetti D.A., Barber A.J., Hollinger L.A., Wolpert E.B., and Gardner T.W. Vascular endothelial growth factor induces rapid phosphorylation of tight junction proteins occludin and zonula occluden 1. A potential mechanism for vascular permeability in diabetic retinopathy and tumors. J Biol Chem, 1999, 274, 23463-23467.

Baumeister U., Funke R., Ebnet K., Vorschmitt H., Koch S., and Vestweber D. Association of Csk to VE-cadherin and inhibition of cell proliferation. Embo J, 2005, 24, 1686-1695.

Behzadian M.A., Windsor L.J., Ghaly N., Liou G., Tsai N.T., and Caldwell R.B. VEGFinduced paracellular permeability in cultured endothelial cells involves urokinase and its receptor. Faseb J, 2003, 17, 752-754.

Carmeliet P., and Jain R.K. Angiogenesis in cancer and other diseases. Nature, 2000, 407, 249-257.

Carmeliet P., Lampugnani M.G., Moons L., Breviario F., Compernolle V., Bono F., Balconi G., Spagnuolo R., Oostuyse B., Dewerchin M., Zanetti A., Angellilo A., Mattot V., Nuyens D., Lutgens E., Clotman F., de Ruiter M.C., Gittenberger-de Groot A., Poelmann R., Lupu F., Herbert J.M., Collen D., and Dejana E. Targeted deficiency or cytosolic truncation of the VE-cadherin gene in mice impairs VEGF-mediated endothelial survival and angiogenesis. Cell, 1999, 98, 147-157.

Cooke V.G., Naik M.U., and Naik U.P. Fibroblast growth factor-2 failed to induce angiogenesis in junctional adhesion molecule-A-deficient mice. Arterioscler Thromb Vasc Biol, 2006, 26, 2005-2011.

Corada M., Zanetta L., Orsenigo F., Breviario F., Lampugnani M.G., Bernasconi S., Liao F., Hicklin D.J., Bohlen P., and Dejana E. A monoclonal antibody to vascular endothelial- 
cadherin inhibits tumor angiogenesis without side effects on endothelial permeability. Blood, 2002, 100, 905-911.

Dejana E., Bazzoni G., and Lampugnani M.G. Vascular endothelial (VE)-cadherin: only an intercellular glue? Exp Cell Res, 1999, 252, 13-19.

Eriksson U., and Alitalo K. Structure, expression and receptor-binding properties of novel vascular endothelial growth factors. Curr Top Microbiol Immunol, 1999, 237, 41-57.

Gavard J., and Gutkind J.S. VEGF controls endothelial-cell permeability by promoting the beta-arrestin-dependent endocytosis of VE-cadherin. Nat Cell Biol, 2006, 8, 12231234.

Gory-Fauré S., Prandini M.H., Pointu H., Roullot V., Pignot-Paintrand I., Vernet M., and Huber P. Role of vascular endothelial-cadherin in vascular morphogenesis. Development, 1999, 126, 2093-2102.

Harhaj N.S., Felinski E.A., Wolpert E.B., Sundstrom J.M., Gardner T.W., and Antonetti D.A. VEGF activation of protein kinase C stimulates occludin phosphorylation and contributes to endothelial permeability. Invest Ophthalmol Vis Sci, 2006, 47, 51065115.

Hashizume H., Baluk P., Morikawa S., McLean J.W., Thurston G., Roberge S., Jain R.K., and McDonald D.M. Openings between defective endothelial cells explain tumor vessel leakiness. Am J Pathol, 2000, 156, 1363-1380.

Hirase T., Staddon J.M., Saitou M., Ando-Akatsuka Y., Itoh M., Furuse M., Fujimoto K., Tsukita S., and Rubin L.L. Occludin as a possible determinant of tight junction permeability in endothelial cells. J Cell Sci, 1997, 110 ( Pt 14), 1603-1613.

Ishida T., Kundu R.K., Yang E., Hirata K., Ho Y.D., and Quertermous T. Targeted disruption of endothelial cell-selective adhesion molecule inhibits angiogenic processes in vitro and in vivo. J Biol Chem, 2003, 278, 34598-34604. 
Kouklis P., Konstantoulaki M., and Malik A.B. VE-cadherin-induced Cdc42 signaling regulates formation of membrane protrusions in endothelial cells. J Biol Chem, 2003, 278, 16230-16236.

Lambeng N., Wallez Y., Rampon C., Cand F., Christe G., Gulino-Debrac D., Vilgrain I., and Huber P. Vascular endothelial-cadherin tyrosine phosphorylation in angiogenic and quiescent adult tissues. Circ Res, 2005, 96, 384-391.

Lampugnani M.G., Zanetti A., Corada M., Takahashi T., Balconi G., Breviario F., Orsenigo F., Cattelino A., Kemler R., Daniel T.O., and Dejana E. Contact inhibition of VEGFinduced proliferation requires vascular endothelial cadherin, beta-catenin, and the phosphatase DEP-1/CD148. J Cell Biol, 2003, 161, 793-804.

Liao F., Doody J.F., Overholser J., Finnerty B., Bassi R., Wu Y., Dejana E., Kussie P., Bohlen P., and Hicklin D.J. Selective targeting of angiogenic tumor vasculature by vascular endothelial-cadherin antibody inhibits tumor growth without affecting vascular permeability. Cancer Res, 2002, 62, 2567-2575.

Liao F., Li Y., O'Connor W., Zanetta L., Bassi R., Santiago A., Overholser J., Hooper A., Mignatti P., Dejana E., Hicklin D.J., and Bohlen P. Monoclonal antibody to vascular endothelial-cadherin is a potent inhibitor of angiogenesis, tumor growth, and metastasis. Cancer Res, 2000, 60, 6805-6810.

Naik M.U., Mousa S.A., Parkos C.A., and Naik U.P. Signaling through JAM-1 and alphavbeta3 is required for the angiogenic action of bFGF: dissociation of the JAM-1 and alphavbeta3 complex. Blood, 2003a, 102, 2108-2114.

Naik M.U., Vuppalanchi D., and Naik U.P. Essential role of junctional adhesion molecule-1 in basic fibroblast growth factor-induced endothelial cell migration. Arterioscler Thromb Vasc Biol, 2003b, 23, 2165-2171. 
Nelson C.M., and Chen C.S. VE-cadherin simultaneously stimulates and inhibits cell proliferation by altering cytoskeletal structure and tension. J Cell Sci, 2003, 116, $3571-3581$.

Nelson C.M., Pirone D.M., Tan J.L., and Chen C.S. Vascular endothelial-cadherin regulates cytoskeletal tension, cell spreading, and focal adhesions by stimulating RhoA. Mol Biol Cell, 2004, 15, 2943-2953.

Rampon C., Bouillot S., Climescu-Haulica A., Prandini M.H., Cand F., Vandenbrouck Y., and Huber P. Protocadherin 12 deficiency alters morphogenesis and transcriptional profile of the placenta. Physiol Genomics, 2008, 34, 193-204.

Shaw S.K., Bamba P.S., Perkins B.N., and Luscinskas F.W. Real-time imaging of vascular endothelial-cadherin during leukocyte transmigration across endothelium. J Immunol, 2001, 167, 2323-2330.

Su W.H., Chen H.I., and Jen C.J. Differential movements of VE-cadherin and PECAM-1 during transmigration of polymorphonuclear leukocytes through human umbilical vein endothelium. Blood, 2002, 100, 3597-3603.

Wallez Y., Cand F., Cruzalegui F., Wernstedt C., Souchelnytskyi S., Vilgrain I., and Huber P. Src kinase phosphorylates vascular endothelial-cadherin in response to vascular endothelial growth factor: identification of tyrosine 685 as the unique target site. Oncogene, 2007, 26, 1067-1077.

Wallez Y., and Huber P. Endothelial adherens and tight junctions in vascular homeostasis, inflammation and angiogenesis. Biochim Biophys Acta, 2008, 1778, 794-809.

Wallez Y., Vilgrain I., and Huber P. Angiogenesis: the VE-cadherin switch. Trends Cardiovasc Med, 2006, 16, 55-59.

Wegmann F., Petri B., Khandoga A.G., Moser C., Khandoga A., Volkery S., Li H., Nasdala I., Brandau O., Fassler R., Butz S., Krombach F., and Vestweber D. ESAM supports 
neutrophil extravasation, activation of Rho, and VEGF-induced vascular permeability.

J Exp Med, 2006, 203, 1671-1677. 